\title{
Small-pad resistive Micromegas for operation at very high rates
}

\author{
M.G. Alviggi ${ }^{a b}$, M. Biglietti ${ }^{c}$, V. Canale ${ }^{a b}$, M. Della Pietra ${ }^{a b}$, C. Di Donato ${ }^{a d}$, E. Farina* \\ ${ }^{e}$, S. Franchino ${ }^{f}$, P. lengo ${ }^{g}$, M. lodice $^{c}$, F. Petrucci ${ }^{c h}$, A. Renardi ${ }^{a b}$, E. Rossi $^{c h}$, G. $^{2}$ \\ Sekhniaidze $^{a}$, O. Sidiropoulou ${ }^{g i}$, V. Vecchio ${ }^{c h}$ \\ ${ }^{a}$ INFN Napoli, Via Cinthia, 80126 Naples, Italy \\ ${ }^{b}$ Universita di Napoli Federico II, Via Cinthia, 80126 Naples, Italy, \\ ${ }^{c}$ INFN Roma Tre, Via della Vasca Navale 84, 00146 Rome, Italy \\ ${ }^{d}$ Universita di Napoli Parthenope, Via Acton 38, 80133 Naples, Italy \\ ${ }^{e}$ Universita di Pavia e INFN, Via Bassi, 6, 27100, Pavia, Italy \\ ${ }^{f}$ Heidelberg University, Grabengasse 1, 69117 Heidelberg, Germany, \\ ${ }^{g}$ CERN, Route de Meyrin 385, 1217 Meyrin, Switzerland, \\ ${ }^{h}$ Universita di Roma Tre, Via della Vasca Navale 84, 00146 Rome, Italy \\ ${ }^{i}$ Bayerische Julius Max. Universitaet Wuerzburg, Sanderring 2, 97070 WuİĹrzburg, Germany \\ E-mail: edoardo.maria.farina@cern.ch
}

\begin{abstract}
We present the development of resistive Micromegas with $\mathrm{O}\left(\mathrm{mm}^{2}\right)$ pad readout aiming at precision tracking in high rate environment without efficiency loss up to few $\mathrm{MHz} / \mathrm{cm}^{2}$. Characterization and performance studies of the detector have been carried out by means of radioactive sources, X-Rays, cosmic rays and test beam data. Gain has been measured as a function of amplification and drift electric fields, under low and high irradiation fluxes. Measurements of the detector efficiency, cluster multiplicity, cluster size and spatial resolution using test beam data will be reported.
\end{abstract}

5th International Conference on Micro-Pattern Gas Detectors (MPGD2017)

22-26 May, 2017

Philadelphia, USA

*Speaker. 


\section{Small-pad resistive Micromegas}

Resistive Micromegas technology has been developed in view of its use in large area detectors for future high energy physics experiment upgrades [1]. It is based on standard Micromegas structure, composed of a drift and amplification gaps [2], and a readout based on parallel strips. The term resistive denotes the presence of a resistive layer, which has been insulated from the readout strips by means of a Kapton ${ }^{\circledR}$ foil. Small prototypes $\left(10 \times 10 \mathrm{~cm}^{2}\right)$ have shown no degradation of the performance in high particle rate environment up to $70 \mathrm{kHz} / \mathrm{cm}^{2}$ [5]. The main obstacles to reach even higher rate are represented by the voltage drop due to the resistive strips and the occupancy increase. However, it is of utmost interest to address the improvement of these two aspects, as future applications will require detectors to sustain particle rate of about $10 \mathrm{MHz} / \mathrm{cm}^{2}$.

A novel prototype has recently been developed having readout and resistive pads in lieu of the commonly adopted strips. A similar approach has been already exploited, as previously reported in [3],[4]. In figure 1, a schematics of the readout PCB is shown. Readout and resistive pads are connected by means of embedded resistors of few $\mathrm{M} \Omega$, giving continuity to the high voltage and evacuating charges. Details concerning the construction of the prototype can be found here [6]. The detector is composed of a matrix of $48 \times 16$ pads, each pad is $1 \times 3 \mathrm{~mm}^{2}$, for a total number of 768 pads. Each pad is an individual readout channel. The drift gap is $5 \mathrm{~mm}$, while the amplification one is of $128 \mu \mathrm{m}$. The active area is $5 \times 5 \mathrm{~cm}^{2}$.

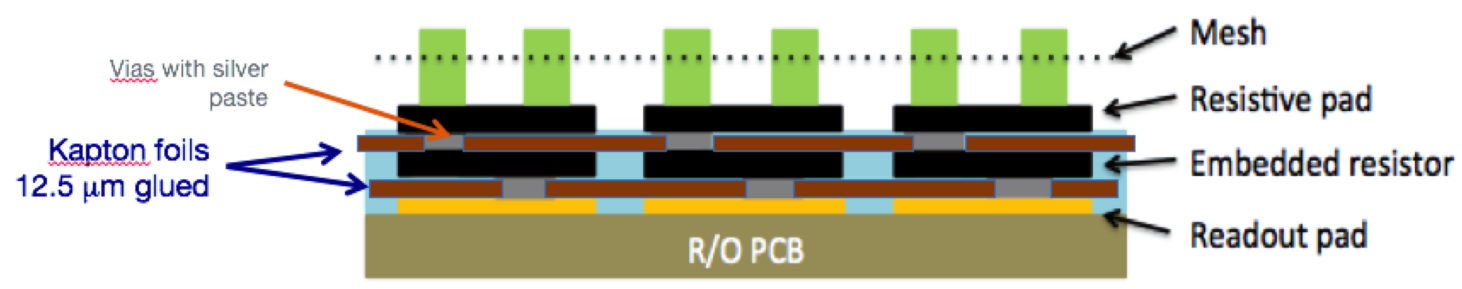

Figure 1: PCB schematics: resistive pads are connected to readout pads by means of embedded resistors of few $\mathrm{M} \Omega$ to evacuate the charges.

\section{Small-pad detector performance}

The characterization of the detector has been carried out by means of X-rays and radioactive sources $\left({ }^{55} \mathrm{Fe}\right)$. The HV was supplied to the drift and the mesh, while the current was read from readout pads. The gas mixture used is $\mathrm{ArCO}_{2}$ (93:7). Figure 2 shows the energy spectrum of the iron source acquired with a Multi Channel Analyser (MCA) for different values of amplification field. An energy resolution of about $30 \%$ has been obtained. This value has been found to be worse than previous results obtained using standard resistive Micromegas detectors. However this might be imputable to inhomogeneities of the field resulting from large border effects due to the highly segmented pad pattern.

The gain of the detector has been measured by means of two different iron sources, one with about $1.3 \mathrm{kHz}$ detected hits, the second about 100 times more $(128 \mathrm{kHz})$. Figure 3 shows the 


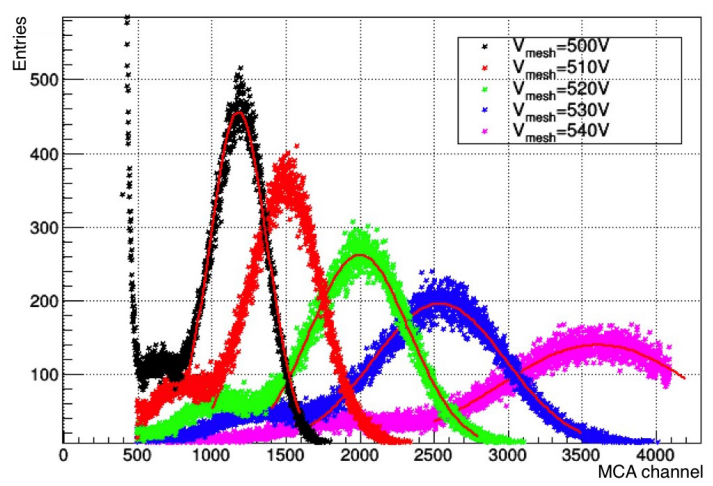

Figure 2: Iron source spectra for different values of the amplification field as recorded with a multi channel analyser (MCA).

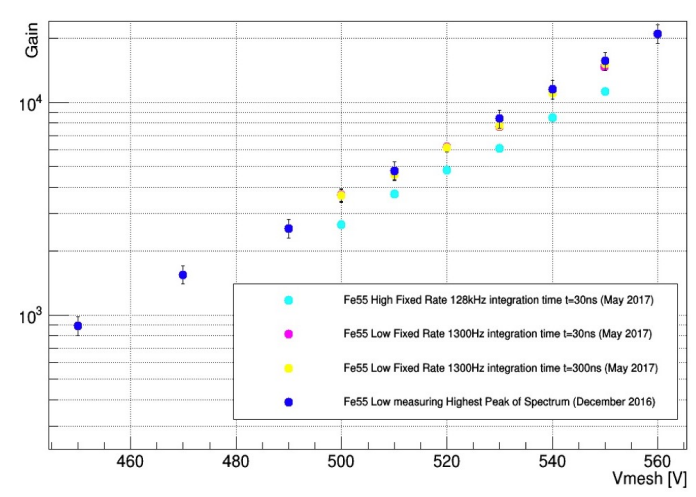

Figure 3: Gain as a function of the amplification voltage for the two iron sources (Low $=1.3 \mathrm{kHz}$, High $=128 \mathrm{kHz}$ ). Comparison among measurements performed at different times is shown.

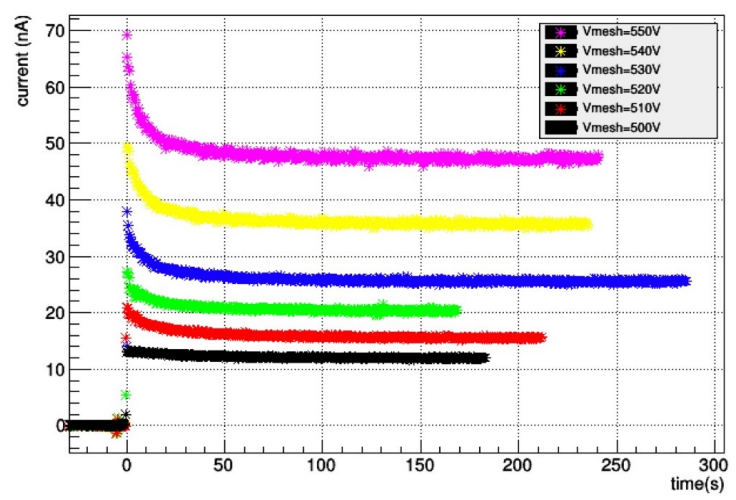

Figure 4: Amplification gain as a function of time as measured in the first seconds after placing the high rate $(128 \mathrm{kHz})$ iron source on the detector.

measured gain as a function of the amplification voltage. A reduction of the gain of about $20 \%$ is observed if the more intense source is used. The dependence of the gain as a function of time has been also studied; figure 4 shows the evolution in time of the current after placing the intense iron source on the detector for few tens of seconds for different amplification fields. As in previous case, a decrease in the gain can be observed: it can be concluded that the charge-up of the dielectric takes place when the intense source is used (higher particle rate), resulting in a local reduction of the amplification field. A saturation behaviour is observed after initial charge-up in about 1 minute.

A systematic study of the gain and of the rate capability has been carried out also with X-rays. The source of photons is a X-Ray gun available at the Gas Detector Development (GDD) laboratory at CERN, providing $8 \mathrm{keV}$ photons from a $\mathrm{Cu}$ anode.

The desired particle rate reaching the detector has been obtained by setting the current of the X-ray gun and by focusing photons by means of $3 \mathrm{~mm} \mathrm{Cu}$ collimator or of an absorber with a 10 $\mathrm{mm}$ hole. Intermediate values for the particle rate have been achieved adding supplementary $\mathrm{Cu}$ attenuators in front of the detector. 
A wide rate range has been explored, while the detector current was constantly monitored. Figure 5 shows the gain as a function of the particle rate for different values of the amplification field. Consistently with what observed with the low/high intensity iron sources, a drop of the gain of about $25 \%$ has been observed. In addition to the charge-up effect, it has also to be considered that the voltage drop due to resistivity of the pads is contributing to the reduction of the gain. With the 3 $\mathrm{mm}$ collimator a rate of about $180 \mathrm{MHz} / \mathrm{cm}^{2}$ has been reached. Figure 6 shows the comparison of the gain as a function of the particle rate for a a fixed value of the amplification field ( $530 \mathrm{~V}$ applied to the mesh) obtained with the two different collimators. A gain of $4 \times 10^{3}$ has been measured at $150 \mathrm{MHz} / \mathrm{cm}^{2}$.

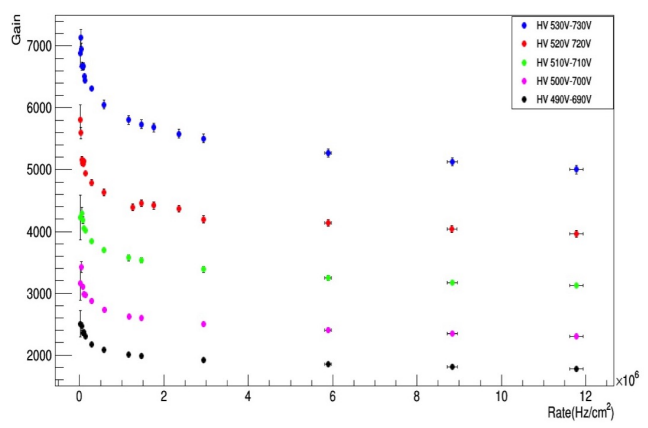

Figure 5: Amplification gain as a function of the rate for different values of the amplification field. A X-ray gun with a $10 \mathrm{~mm}$ hole absorber has been used for the measurements.

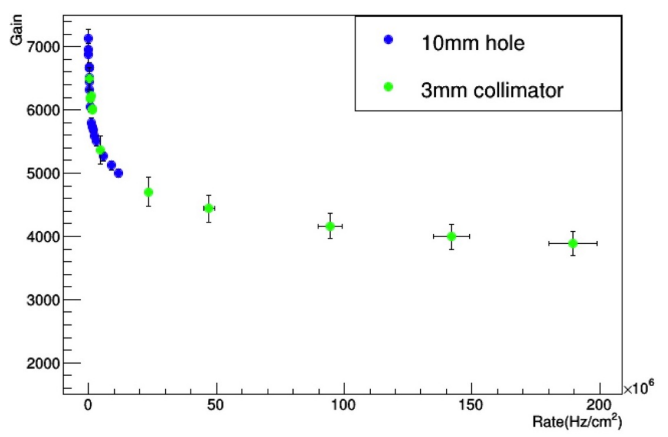

Figure 6: Gain as a function of the rate for a fixed amplification value. A X-ray gun with a $10 \mathrm{~mm}$ hole (blue points) or a $3 \mathrm{~mm}$ collimator (green points) have been used for the measurements.

\subsection{Performance at testbeam}

Detector performance, including efficiency, tracking capability and resolution have been studied in a dedicated testbeam campaign at SPS facility at CERN, where high energy muon and pion beams are available. Data have been acquired with APV-25 hybrids and RD51 SRS system [7]. Double view bulk Micromegas detectors have been used as tracking chambers. The clusters on the resistive pad detector have been reconstructed combining the information from neighbouring fired pads. The resolution has been evaluated comparing the cluster position reconstructed on the detector and the expected hit position obtained from tracking detectors, and then subtracting uncertainty on the track interpolation. Figure 7a shows the residual distribution for the most accurate 
coordinate, corresponding to the short side of the pads, equal to $1 \mathrm{~mm}$. A resolution of $190 \mu \mathrm{m}$ has been obtained. For what concerns the less accurate coordinate, corresponding to the $3 \mathrm{~mm}$ side of the pads, a resolution of $800 \mu \mathrm{m}$ has been found.

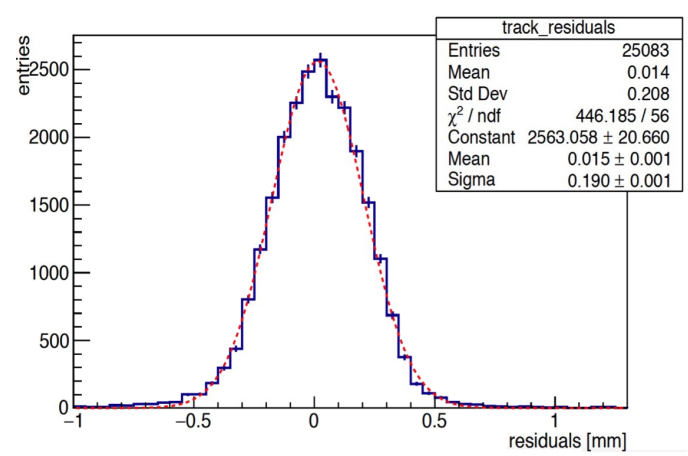

(a)

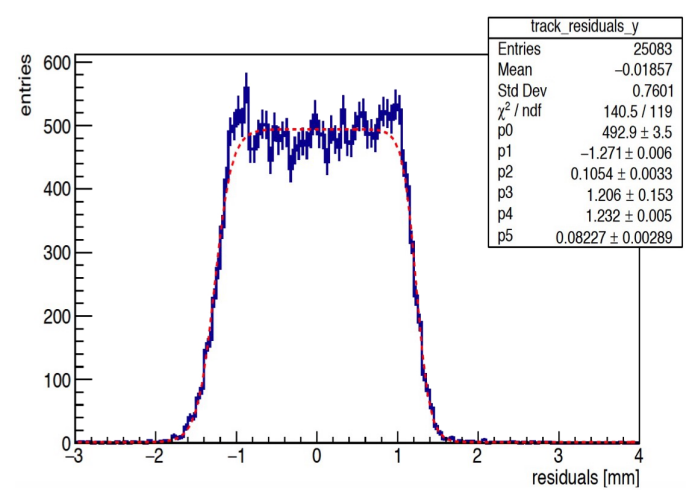

(b)

Figure 7: Residual distribution between expected and reconstructed cluster position for precise coordinate (7a) and second coordinate (7b)

Figure 8 shows the efficiency obtained as a function of the amplification field. An event is defined efficient if a cluster on the pad detector is found within 3 times the resolution of the accurate coordinate around the expected position. The efficiency bends toward a plateau of around $98 \%$. The red points show instead the trend of the efficiency when the whole chamber is considered.

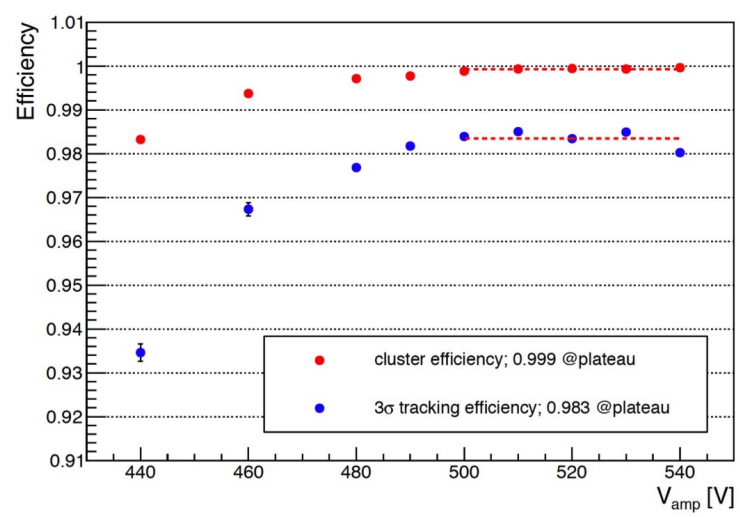

Figure 8: Cluster efficiency as a function of the amplification field. The red points show the efficiency when clusters are selected in all the detector: the plateau reaches about the $99.9 \%$. The blue points represent instead the efficiency obtained by selecting clusters lying not further than 3 times the resolution of the accurate coordinate $(3 \sigma)$ from the expected position. In this case, the plateau reaches the $98 \%$.

As a final remark, the number of fired pads in a cluster has been found to depend on the position of the particle traversing the detector. If the track crosses a pad close to its center, a higher probability to generate a cluster made of an odd number of pads has been observed, as shown in figure 9a. On the other hand, if the track passes close to the edge of a pad (as limit case, exactly 
in between two pads), an higher probability to reconstruct a cluster having an even number of fired pads has been measured (figure $9 b$ ).

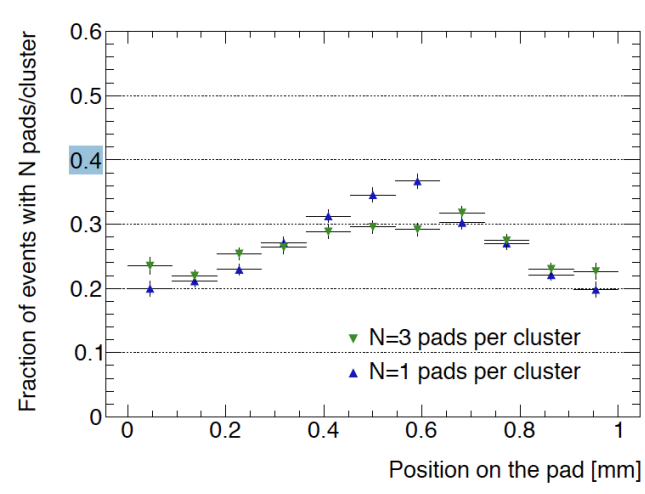

(a)

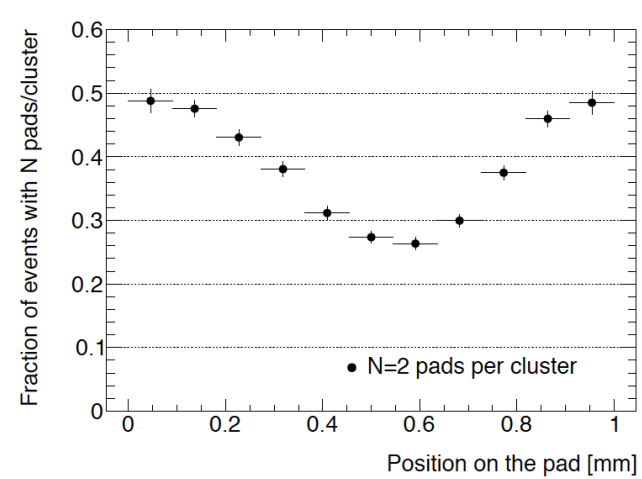

(b)

Figure 9: Probability of firing an odd (a) and even (b) number of pads per cluster as a function of the position of the extrapolated track in the pad.

\section{Conclusions and future prospectives}

A Micromnegas prototype based on resistive pads has been built at CERN, designed to be suitable for high particle rate environment (up to $10 \mathrm{MHz} / \mathrm{cm}^{2}$ ). A complete characterization of the detector, by means of radioactive sources, $\mathrm{X}$-rays and muon beam has been carried out, confirming an excellent rate capability (tested up to $150 \mathrm{MHz} / \mathrm{cm}^{2}$ ) and good resolution $(190 \mu \mathrm{m})$. It is, however, difficult to imagine scaling this design to large area detectors due to complex routing. A new prototype is under construction, with APV25 electronics directly integrated on the PCB.

\section{Acknowledgements}

We would like to thank the CERN MPT Workshop (R. De Oliveira and A.Teixeira for ideas, discussions and construction of the detectors), the RD51 Collaboration for support with tests at the GDD Lab and at H4 SPS beam line.

\section{References}

[1] T. Alexopoulos et al., Nucl. Instrum. Meth. A 640 (2011) 110.

[2] Y. Giomataris et al., Nucl. Instrum. Meth. A 376 (1996) 29-35.

[3] C. Adloff et al., Nucl. Instrum. Meth. A 729 (2013) 90-101.

[4] F. Thibaud at al., JINST 9 (2014) C02005.

[5] 0. Sidiropoulou, et al., Nuclear Instruments and Methods in Physics Reasearch A, 10.1016/j.nima 2016.06.062

[6] M.Alviggi et al., Small-pads resistive micromegas, 2017 JINST 12 C03077

[7] S. Martoiu et al., JINST 8 (2013) C03015 